\title{
Arc-Heater Facility for Hot Hydrogen Exposure of Nuclear Thermal Rocket Materials
}

\author{
Ron J. Litchford ${ }^{1}$, John P. Foote ${ }^{2}$, Ten-See Wang ${ }^{3}$, Robert Hickman ${ }^{4}$, Binayak Panda ${ }^{5}$, \\ Chris Dobson $^{6}$, Robin Osborne ${ }^{7}$, and Scooter Clifton ${ }^{8}$ \\ NASA Marshall Space Flight Center
}

\begin{abstract}
A hyper-thermal environment simulator is described for hot hydrogen exposure of nuclear thermal rocket material specimens and component development. This newly established testing capability uses a high-power, multi-gas, segmented arc-heater to produce hightemperature pressurized hydrogen flows representative of practical reactor core environments and is intended to serve as a low cost test facility for the purpose of investigating and characterizing candidate fuel/structural materials and improving associated processing/fabrication techniques. Design and development efforts are thoroughly summarized, including thermal hydraulics analysis and simulation results, and facility operating characteristics are reported, as determined from a series of baseline performance mapping tests.
\end{abstract}

\section{Introduction}

NUCLEAR thermal propulsion is often suggested as a component of future space transportation architectures because of the potential performance gains over chemical propulsion systems, whose energy content is physically limited by molecular bond strengths. In principle, high-thrust nuclear thermal rockets (NTR) using pure hydrogen propellant could produce twice the specific impulse of chemical rockets and could therefore greatly expand lunar exploration capability and reduce the duration of crewed Mars expeditions to a biologically manageable level. However, previous NTR development efforts have revealed that it is exceedingly difficult to attain this level of performance in practice due to the fundamental material issues and engineering constraints encountered in high-temperature hydrogen environments. The principle design dilemma derives from the need to maximize performance by increasing fuel operating temperature whereas engine durability and reliability is critically dependent on the ability of the fuel element and thrust chamber materials to withstand severe chemical attack by high-pressure hot hydrogen flow.

Although most space missions of interest would at most require only a few hours of solid-core NTR operation, the desire to push fuel element temperatures to extremes in order to maximize performance greatly intensifies hydrogen induced corrosion rates, which are known to increase in direct proportion to reactor operating temperature. As a result, design closure becomes problematic, and the maximum fuel operating temperature must reflect a balance between competing engineering interests, as illustrated on a typical NTR pressure-temperature operating map in Fig. 1. The critical technical obstacle to a practically useful NTR engine, therefore, is the development of innovative high-temperature fuel/structural materials that can endure 2500-3000 K hydrogen environments with minimal corrosion while also possessing sufficient strength and resilience to resist breakage from intense vibration loads and thermally induced stresses.

\footnotetext{
${ }^{1}$ Project Principal Investigator, Thermal/Combustion Devices, Propulsion System Dept., Associate Fellow AIAA.

${ }^{2}$ Project Chief Engineer, Thermal/Combustion Devices, Propulsion Systems Dept.

${ }^{3}$ Technical Assistant, Thermal/Combustion Analysis Branch, Propulsion Systems Dept., Senior Member AIAA.

${ }^{4}$ Materials Engineer, Materials and Processes Laboratory.

$r^{5}$ Materials Engineer, Materials and Processes Laboratory.

${ }^{6}$ Project Physicist, Environmental Test Branch, Test Laboratory.

${ }^{7}$ Project Engineer (ERC Incorporated), Thermal/Combustion Devices, Propulsion Systems Dept.

${ }^{8}$ Facility Engineer (Lockheed-Martin Corp.), Materials Environment Test Complex (METCO).
} 
During the ROVER/NERVA program, considerable progress was made in reactor fuel development yielding a viable technology base of continuing relevance to space exploration needs. Through a development cycle extending over numerous hot reactor tests, the program arrived at coated graphite-based fuel formulations exhibiting good structural integrity and low corrosion rates under sustained high temperature operation (in excess of $2200 \mathrm{~K}$ for more than 1 hour). This significant achievement still falls far short of ultimate material limits, however, and substantial potential exists for further improvement. Moreover, the significant work accomplished by this program is decades old and the resulting knowledge, although summarized in various reports, was never completely encapsulated and transferred to modern generations.

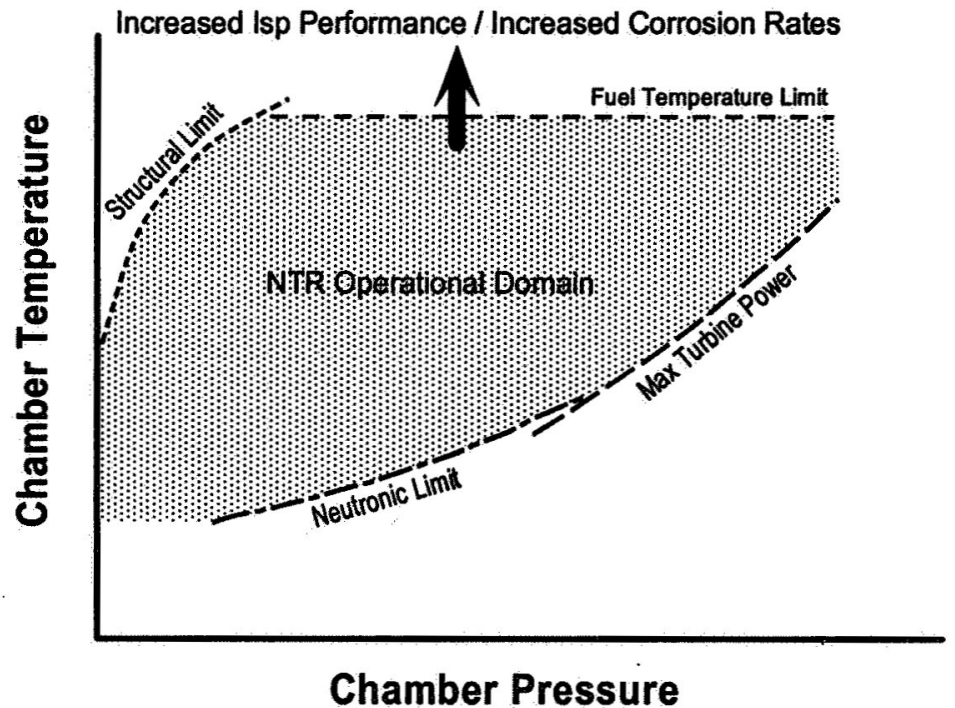

In view of the limited knowledge on phase relationships and stability of these carbon systems as well as the significant advances in high temperature fuel formulations and processing over the intervening years, it is therefore important that reactor fuel technology be revisited and thoroughly re-examined prior to embarking on a modern NTR engine development path. Promising contemporary approaches for realizing additional extensions in operating temperature and lifetime include utilization of modern coating techniques and advanced high-temperature materials such as bi/tri-carbides of the U$\mathrm{Zr}-\mathrm{X}$ type, carbon-nitrides of $\mathrm{U}$ and $\mathrm{Zr}$, and CERMETs based on a $\mathrm{W}$ matrix.

For structural component applications, it would also be desirable to develop a more thorough understanding of refractory metals and their alloys, such as W, Re, W-Re, Mo, Mo-Re, and W-Re-Hfc, because of their high melting temperatures and inherent resistance to hot hydrogen. The application potential of refractory metals is normally constrained, since they usually must be forged to obtain adequate properties and because they suffer from poor ductility and reduced strength at elevated temperatures. Forging refractory metals into complex shapes, however, is difficult and expensive, and several new forming processes have been developed in recent years to overcome these limitations. Some recent innovations relevant to NTR needs include the Vacuum Plasma Spray (VPS) and Electroforming (El-Form) processes, which were developed for fabricating low cost, net shape refractory metal components such as boost and tactical motor nozzles.

Practical development and characterization of these advanced fuel/structural materials would greatly benefit from low-cost non-nuclear test and evaluation methods in which material samples could be exposed to severely corrosive hot hydrogen environments with temperatures ranging as high as $3000 \mathrm{~K}$. Due to the hazards associated with hot hydrogen and the limited scope of utilization, laboratory facilities for this type of R\&D work are not generally available and special efforts are needed to develop and establish this class of non nuclear test capability. This paper describes the development of an NTR environment simulator based on a high-power multi-gas arc-heater thermal source, which was commissioned for the purpose of partially satisfying this critical need.

\section{Technical Approach}

A suggested technical approach for non-nuclear NTR materials development is summarized in the process diagram of Fig. 2. The critical R\&D strategy is represented by the inner iterative loop, which focuses on developing and improving innovative processing/forming techniques for NTR fuels and components. Two complimentary test capabilities were envisioned to support this strategy as indicated on the diagram. The first would rely on modification of an existing multi-gas high-power arc-heater facility for hydrogen operation and development of an attachable test fixture for exposure of small material specimens, as illustrated in Fig. 3. A second more sophisticated approach would rely on the build-up of an RF-heated fuel element simulator, which would provide for more accurate simulation of reactor environments. The focus of this project was the arc-heater based approach since it could be developed at lower cost and could be pressed into useful operation on an accelerated schedule. 


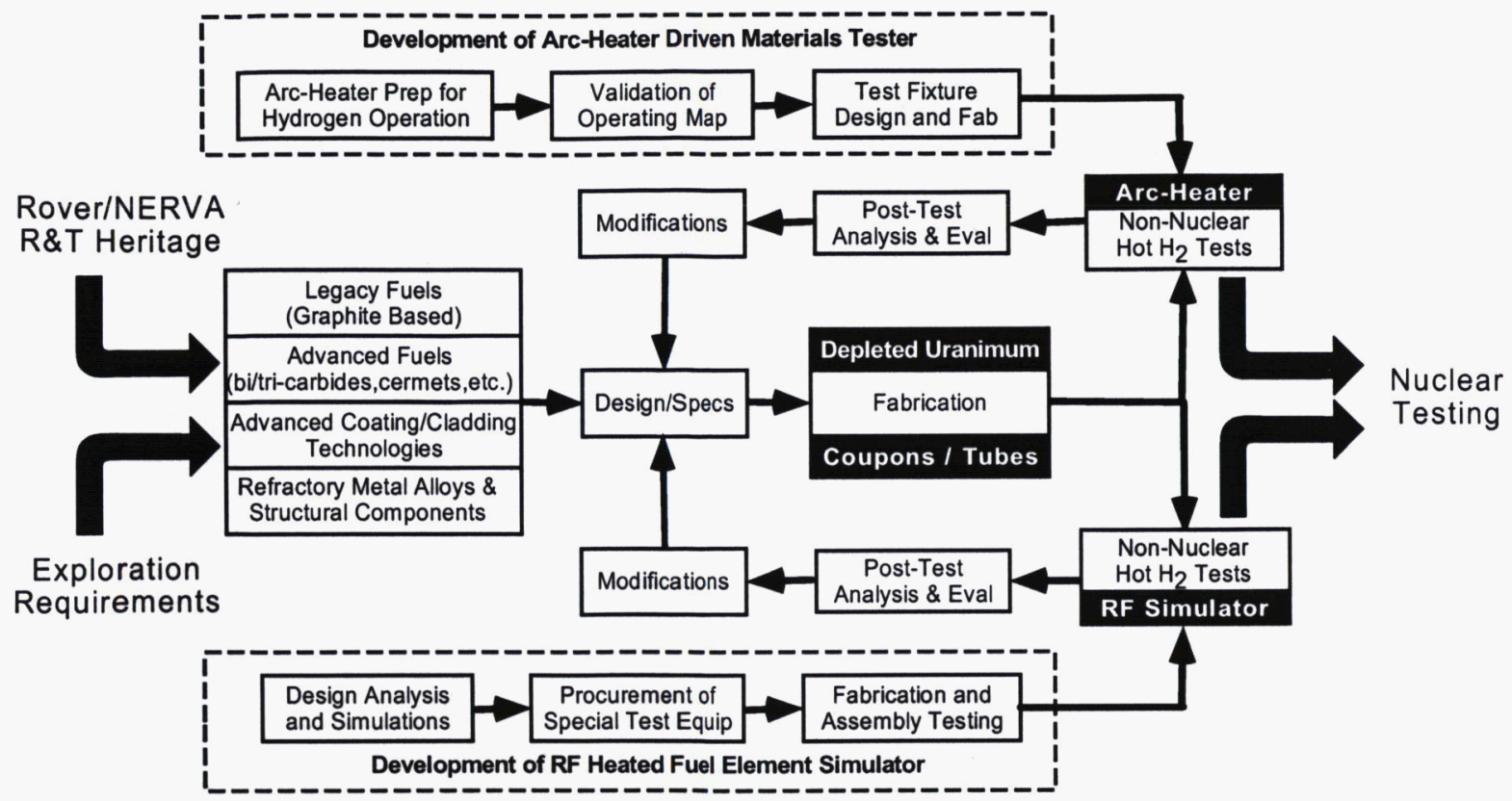

Figure 2. Non-nuclear research strategy for hot hydrogen materials and component development.

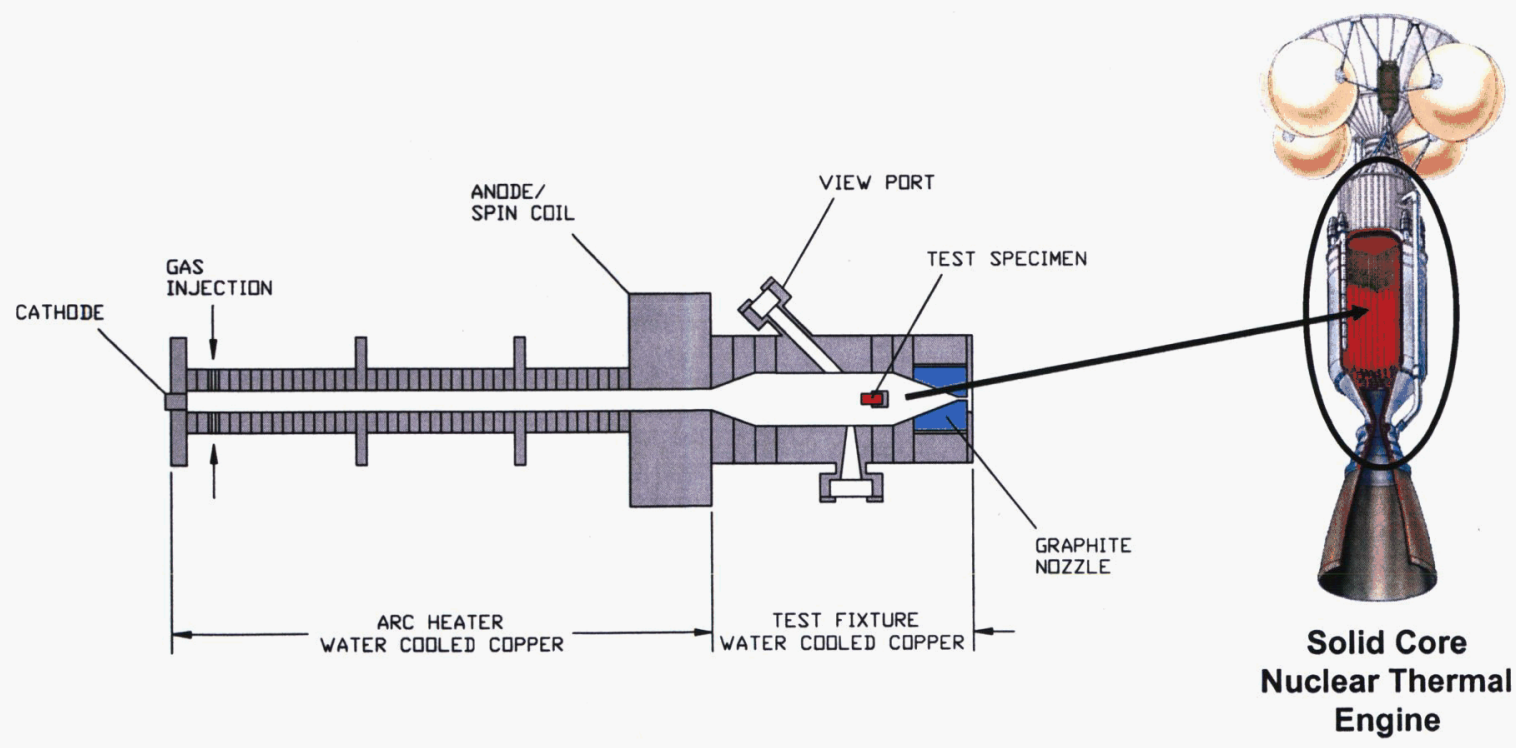

Figure 3. Arc-heater based nuclear thermal rocket environment simulator for the generation of hyperthermal convective conditions traceable to reactor core environments

The intent of the suggested non-nuclear R\&D approach is to enable rapid screening and assessment of candidate material specimens, at relatively low cost, and to identify those methods worthy of more in-depth study and systematic development. The basic idea is to produce small, easily fabricated material specimens using innovative processing and manufacturing techniques and to expose these specimens to relevant hot hydrogen environments, excluding radiation effects. Materials and processing techniques could then be perfected by following an iterative approach whereby understanding and knowledge gained from post-test analysis and inspections of exposed specimens are used to validate physics based modeling efforts and to improve fabrication/processing techniques. It is believed that this type of non-nuclear approach will be the most cost effective strategy for arriving at optimal material candidates for actual nuclear environments testing, which can be orders of magnitude more expensive to conduct. A peripheral but important secondary objective of this project is the detailed measurement of hot hydrogen 
thermodynamic properties and their effect on hydrogen engine performance since available data are not firmly anchored to reliable empirical measurements in the temperature regime of interest. The technological knowledge base established through such efforts would represent a valuable contribution to long term space exploration goals and would provide a solid foundation for future development of an NTR flight engine.

\section{Facility Development}

\section{A. Arc-Heater Description}

The hyper-thermal conditions for simulation of NTR reactor core environments are generated by a multi-gas high-power segmented arc-heater, which is capable of processing large hydrogen flow rates in excess of $3000 \mathrm{~K}$. This device was originally developed by Aerotherm Corporation in Mountain View, CA more than 30 years ago, but was decommissioned and transferred to Marshall Space Flight Center in 1998 with the intent of supporting propulsion materials development and qualification testing, particularly solid motor nozzle materials.

The 1-inch bore arc-heater consists of 3/8-inch thick copper segments stacked in a three-pack configuration yielding an overall length of about 1-meter. The working gas is injected tangentially through four 0.048 -inch jets in a Primary Gas Injection (PGI) segment near the rear of the arc-heater, and a DC arc discharge is established between a tungsten cathode button in the rear sealing flange and a copper anode ring at the arc-heater exhaust. A magnetic spin coil is located around the anode ring to induce continuous rotation of the arc attachment point. In order to start the device at atmospheric chamber pressure, a modest flow of argon is first introduced into the arc-heater and an initial gas discharge is established between the cathode button and a starting segment, which is located just downstream of the PGI segment. The anode power lead is then automatically switched from the starting segment to the anode ring in order to establish a stable arc down the full length of the arc-heater. At this point, argon flow can be replaced with the desired working gas and the system can be ramped to the desired operating state.

The current system power supply permits sustained operation at $0.75-\mathrm{MW}_{\mathrm{e}}$ and short duration runs at $1.5-\mathrm{MW}_{\mathrm{e}}$ for several minutes. Because NTR burn times are on the order of 1 to 2 hours for most space missions of interest, the nuclear thermal environment simulator facility was designed to provide a continuous flow of pressurized hot hydrogen over a material specimen using a maximum electrical input power of $750 \mathrm{~kW}_{\mathrm{e}}$. The achievable hydrogen flow rates over the desired temperature range were estimated using the NASA Chemical Equilibrium Code (CEC) assuming various values for the electric-to-thermal efficiency. The results are summarized in Fig. 4 for 300, 400, and $500 \mathrm{~kW}_{\text {th }}$ of thermal input power for chamber pressures of 11,18 , and $35 \mathrm{~atm}$. Historical data on arc-heater performance indicated an expected efficiency in the range of 50 to 60 percent for these conditions, which implied a hydrogen flow rate of 7 $-10 \mathrm{~g} / \mathrm{s}$ over a temperature range of $2500-3500 \mathrm{~K}$. Pressure effects were found to have only a minor perturbative effect within this temperature range.

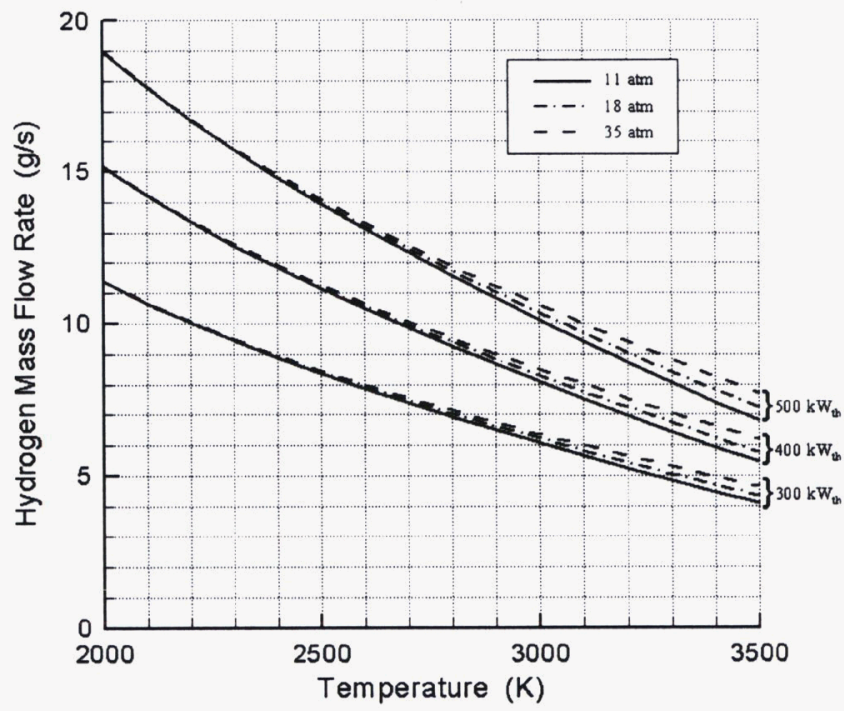

Figure 4. Estimated hydrogen heating characteristics assuming $750 \mathrm{~kW}_{\mathrm{e}}$ input power and various values for electric-to-thermal conversion efficiency.

\section{B. Test Fixture Design and Analysis}

A modular water-cooled test fixture was designed in which small material specimens could be securely mounted and exposed to the arc-heated hydrogen flow through direct attachment to the arc-heater exhaust flange. The test fixture design also included optical access features for real-time pyrometer measurement of specimen surface temperature and for non-intrusive laser-based measurement of the hydrogen thermodynamic state. The baseline design specifications for the test fixture are summarized in Table 1.

Table 1. Test Fixture Design Specifications.

\begin{tabular}{ll}
\hline \hline$T_{\max }$ & $3500 \mathrm{~K}$ \\
$p_{\max }$ & $35 \mathrm{~atm}$ \\
$\dot{m}_{\text {hydrogen }}$ & $5-10 \mathrm{~g} / \mathrm{s}$ \\
$d_{\text {bore }}$ & 2.5 inches \\
$d_{\text {specimen }}$ & 0.5 inches \\
$l_{\text {specimen }}$ & 1.0 inches \\
\hline
\end{tabular}




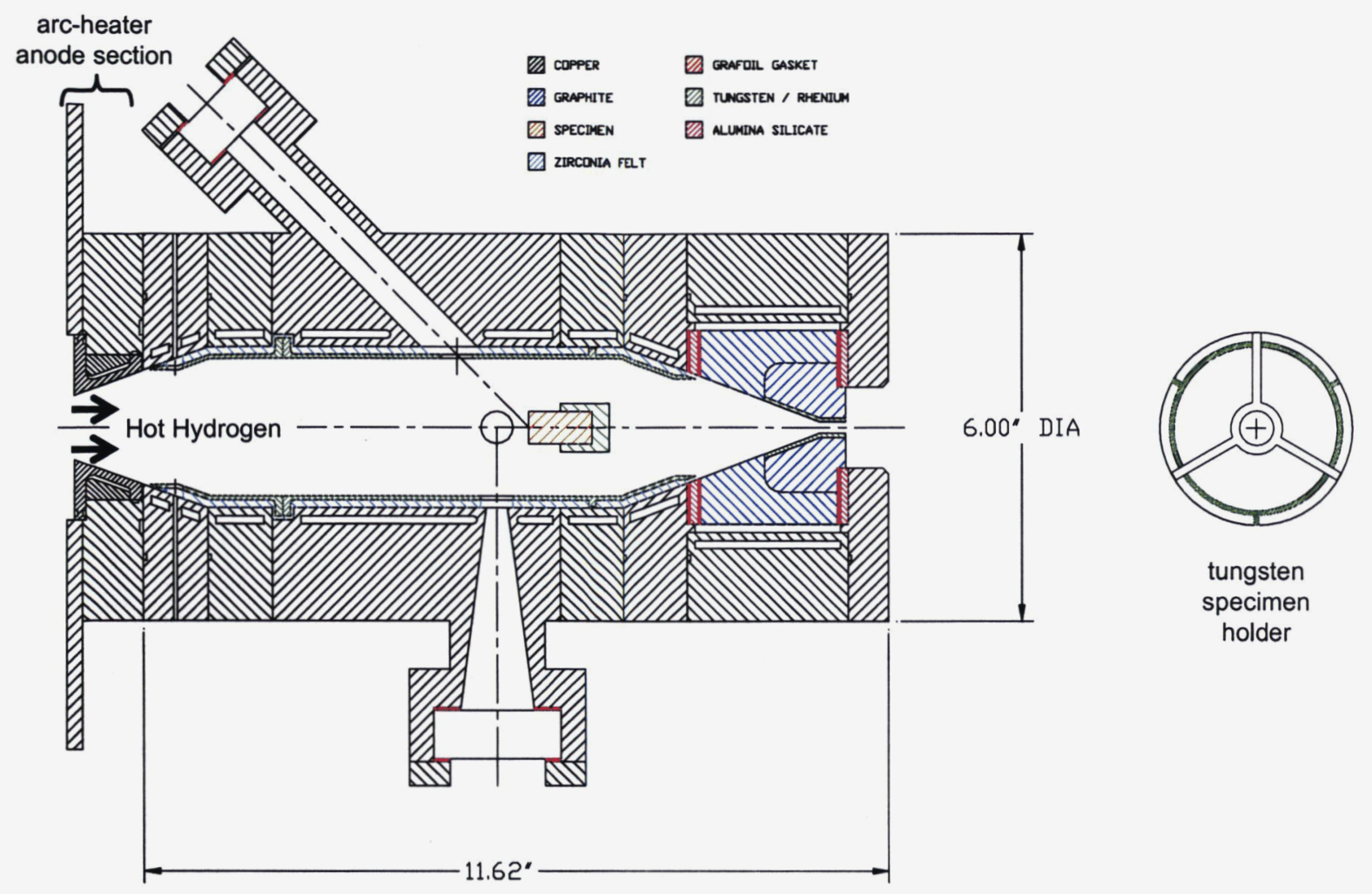

Figure 5. Test fixture layout drawing for the hot hydrogen nuclear thermal environments simulator.

A detailed mechanical design for the test fixture was developed based on these specifications, yielding the final layout shown in Fig. 5. The test fixture is essentially configured as a stack of water-cooled copper segments including an adaptor attachment to the arc-heater anode section and a converging nozzle section where the flow is throttled through a graphite nozzle with a tungsten throat insert. The mid-section contains features for insertion of a tungsten spider to hold small material specimens (1-inch long, 1/2-inch diameter rods) and has four windowed ports for optical access. One port is canted to allow pyrometer measurements of specimen surface temperature during exposure. Two diametrically opposed ports allow for focusing of a laser excitation beam at a location just upstream of the specimen nose, and a fourth port is rotated $90^{\circ}$ out of this plane for capture of Raman scattering from diatomic hydrogen, which can be used to infer the rotational temperature and number density of molecular hydrogen.

Externally, the test fixture has a length of about 12 inches and a diameter of about 6 inches. Because all cooling water is drawn from a single 350-psig supply manifold, the water passages were sized to have the same pressure drop as the arc-heater segments in order to obtain proper flow rate splitting. This required the milling of $1 / 8$-inch passages into the copper segments, which were sealed with e-beam welded ring plates. Stress considerations for the 2.5-inch inner bore diameter required a 1/8-inch thickness between the water passages and the inner wall surface. These dimensions may be compared with 1/16-inch passages and 1/16-inch wall thicknesses for the arc-heater segments, which have a 1-inch bore diameter.

Thermal hydraulic analyses were performed using an in-house three-dimensional computational fluid dynamics tool (UNIC) to assist in test fixture design and performance assessment. Multi-physics invoked in these analyses include turbulence, hydrogen dissociation, equilibrium thermodynamics, convective heat transfer, and radiation transport. The main goals of this supporting analysis was to quantify chamber wall heat flux and combined heat transport effects on specimen temperature so that the desired exposure conditions could be achieved during materials development tests.

Initially, we considered a basic design configuration in which there was no thermal barrier between the hot hydrogen flow and the copper wall. Detailed analyses of this configuration revealed the formation of intense vortices near the wall which raised the local heat flux beyond acceptable limits for the maximum specified operating conditions. Moreover, it was observed that radiation losses from the specimen to the cold copper wall through the highly transparent hydrogen were draining energy from the specimen and significantly lowering the surface temperature below the gas temperature. To circumvent these design problems, a 0.060 -inch thick $\mathrm{W} / 5 \% \mathrm{Re}$ heat 
shield was inserted with a 0.125 -inch gap between the shield and the copper wall, as shown in the layout drawing of Fig. 5. Dams were also incorporated into the shield design to prevent seepage of hot hydrogen flow through the backside gap. Detailed analyses of this configuration showed that the high heat flux vortices were completely isolated on the inner surface of the shield and revealed that the wall heat flux could be kept below an acceptable $500 \mathrm{~W} / \mathrm{cm}^{2}$ at the maximum design operating temperature. The computed temperature contours, atomic hydrogen concentration contours, and streamlines are shown in Fig. 6 for the finalized test fixture layout.

From the temperature contours, it can be seen that the flow is uniformly hot and that the hydrogen jet impinges and wraps around the specimen with minimum energy dissipation. The atomic hydrogen concentration between the chamber and the shield is lower because of the reduced convective heat transfer. The streamlines show that the high inlet flow speed generates a small recirculation bubble at the divergent section, which allows the central sample surface temperature to attain a very high value (i.e., $3426.0 \mathrm{deg}$. $\mathrm{K}$ accounting for radiation).

Thermal and stress design analyses were performed in-house, and the final working drawings were reviewed and approved prior to letting of contracts for component fabrication.

Photographs of fabricated test fixture hardware and refractory metal alloy components are shown in Fig. 7. The tungsten specimen holder was machined from a tungsten block using Electrical Discharge Machining (EDM) methods and is shown with a W-carbide specimen, which is secured to the holder using tungsten pins. The tungsten nozzle insert was also machined using EDM methods. The large diameter W/5\%Re heat shield was formed as a net shaped part using a Vacuum Plasma Spray (VPS) technique and the outer surface was ground finished. This particular part is quite unique in terms of material, size, and shape and represents a fundamental advancement in VPS fabrication capability.

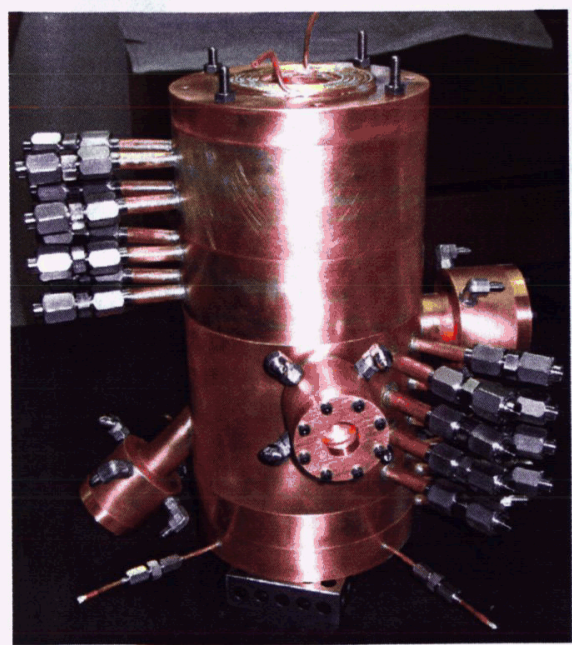

Test Fixture Assembly

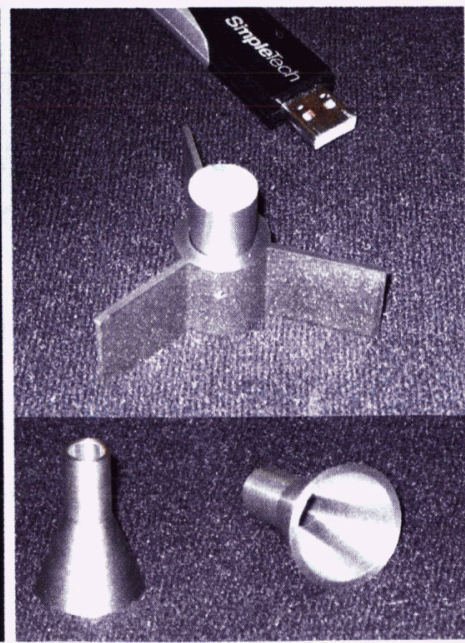

Specimen Holder \& Nozzle Insert

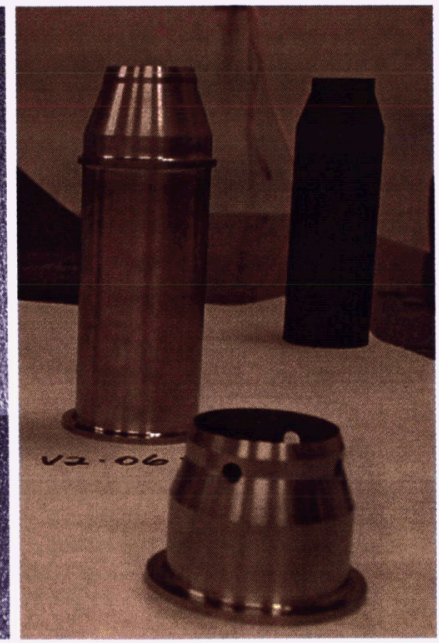

W/5\%Re Heat Shield

Figure 7. Photographs of fabricated test fixture hardware and refractory metal alloy components. 


\section{Arc-Heater Performance Characterization}

As the various test fixture pieces and components were being fabricated, parallel project efforts were directed at establishment of basic arc-heater operational capability for hydrogen. This included installation of a supply line with a flow meter and regulator sized for delivery of $10 \mathrm{~g} / \mathrm{s}$ of gaseous hydrogen from a high-pressure, high-capacity tube trailer. Flow rate estimations indicated that a fully charged tube trailer would have sufficient capacity for more than 1-hour of continuous arc-heater operation. As part of this general activation activity, it was also necessary to refurbish and assemble various facility components, establish and implement operational procedures, and conduct thorough safety/hazard assessments.

Baseline arc-heater performance capability was established by attaching a sacrificial graphite nozzle assembly directly to the arc-heater anode flange. The sacrificial nozzle was machined from high-density graphite with a throat diameter matching the final test fixture design specifications so that the proper flow rate and chamber pressure would be produced at the specified mass flow rate and input power settings. This approach provided a reliable method for accurate performance characterization while test fixture hardware was in fabrication.

An arc-heater starting sequence was successfully established for hydrogen operation, and we then embarked on a series of performance mapping tests to empirically correlate operational parameters with the controllable input settings. In this case, the two controllable input parameters were the applied arc current and the mass flow rate, as established by the supply pressure to the PGI jets. The applied voltage of the power supply was uniquely determined by the arc plasma impedance for the applied current setting.

After a few exploratory tests, we were able to verify steady-state operational conditions could be reliably established within 20 seconds of arc initiation, as may be seen from the start-up transients for a typical 60 -second hydrogen run in Fig. 8. Therefore, we were able to generate steadystate performance tests with run times restricted to the range of $30-60$ seconds.

During these test, we recorded basic operating parameters such as current, voltage, chamber pressure, hydrogen mass flow rate, cooling water temperature rise, and cooling water flow rate from which we could infer system power balance and electrical-to-thermal conversion efficiency. The thermal input power, for example, was defined as the difference between the electrical input power and the heat loss power to the cooling water, and this parameter was then used to infer the efficiency. Given the thermal power input and the known throat size, we could also estimate the total enthalpy/temperature of the hydrogen in the chamber by running a rocket calculation using the NASA Chemical Equilibrium Code (CEC). The results obtained using this particular approach appeared to be very reliable since the predicted chamber pressure differed from the measured value by less than 1 percent in all cases. Although the gas is clearly in a highly non-equilibrium state within the instantaneous arc region, the volume of this region is small in comparison to the bulk flow and the rapidly fluctuating arc structure insures intense mixing of the two regions.
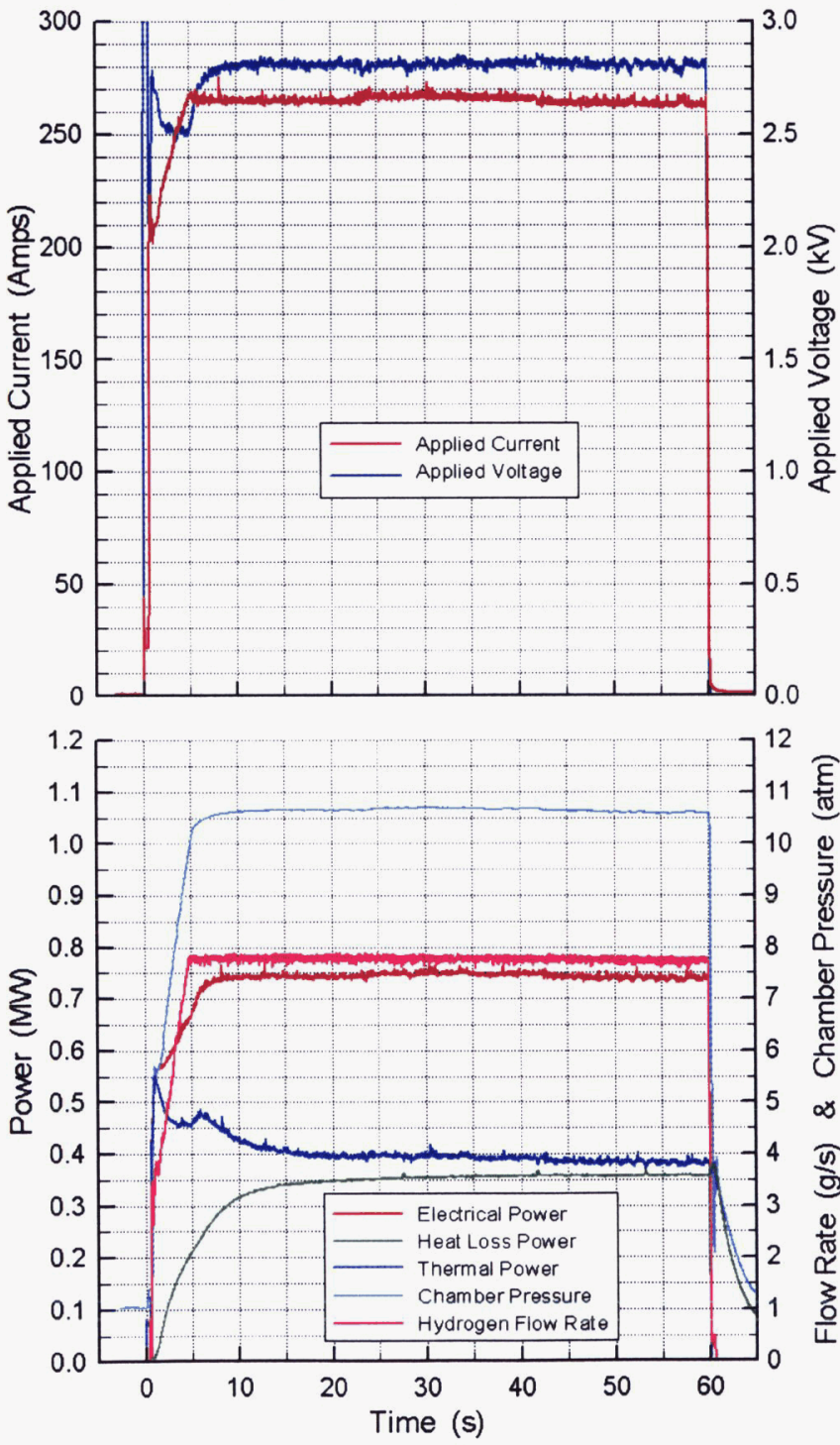

Figure 8. Start-up transients and establishment of steadystate operating conditions during a typical arc-heater performance mapping test. 

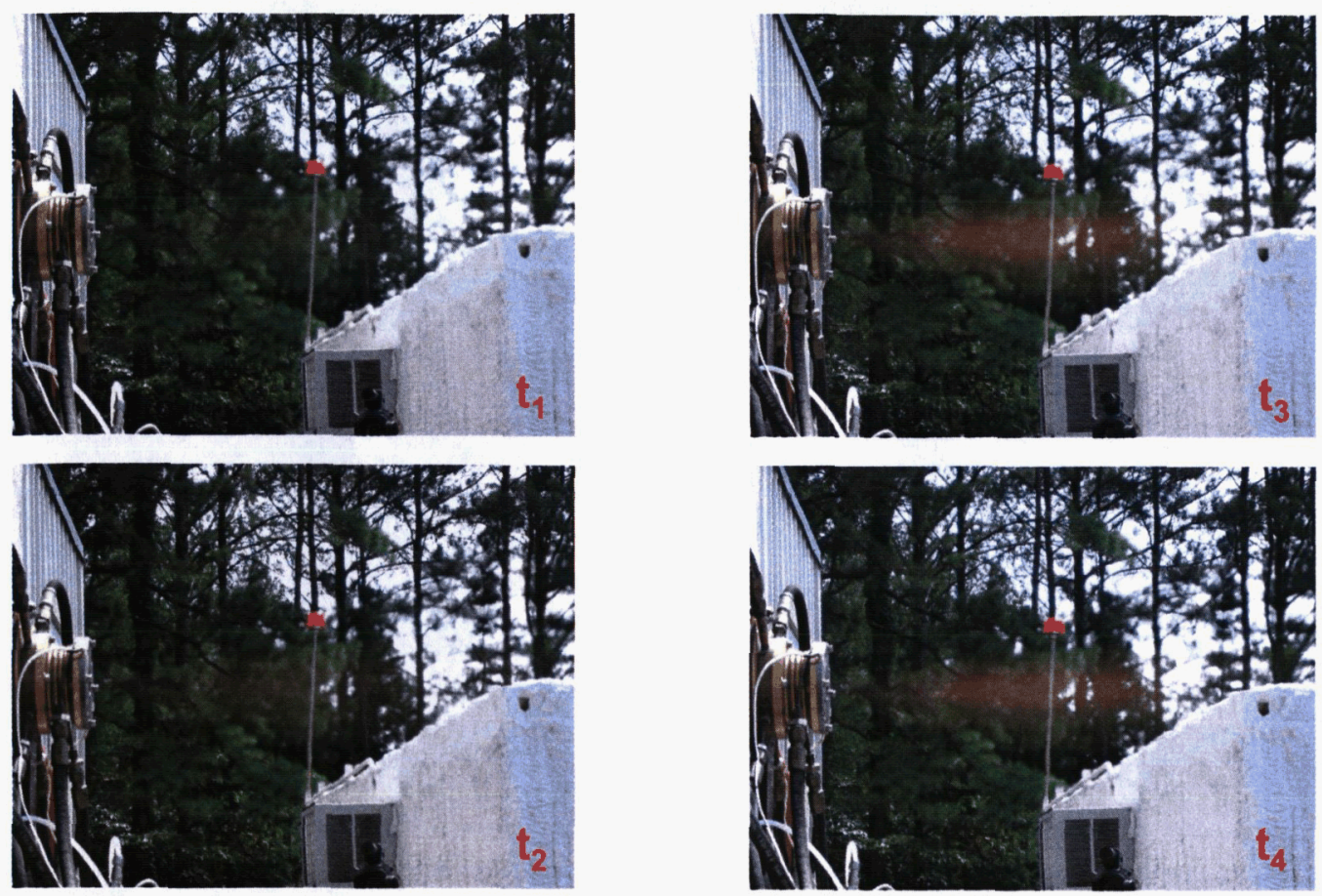

Figure 9. Time sequenced plume images during typical performance mapping run $\left(t_{1}<t_{2}<t_{3}<t_{4}\right)$. Note delayed appearance of soot emission from methane after-burning as graphite nozzle heats to steady state temperature.

About 80 runs were successfully completed during the performance characterization test program, which yielded a database sufficiently populated to support the development of a reliable operating map. The integrated run times for hydrogen operation exceeded 40 minutes, and the uncoated graphite throat eroded from 0.273 " $\varnothing$ to 0.279 " $\varnothing$ over that entire period. It should be pointed out that graphite erosion was least severe during the initial 30 seconds of operation and significantly more severe following attainment of the fully thermalized state, as may be observed from the time sequenced plume images of Fig. 9. The hot hydrogen plume remained essentially transparent during the initial warm-up stage, but after about 30 seconds of operation the graphite nozzle finally attained thermal steady state and soot formation could thereafter be observed as a result of methane after-burning.

The operating load lines for hydrogen operation are summarized for four different flow rates in Fig. 10. For applied currents between 200 and 300 amps, the load impedance was in the range of 2500 to 3000 volts as the hydrogen flow rate was varied between 7.0 and $10.4 \mathrm{~g} / \mathrm{s}$. Applied currents less than about 200 amps yielded unstable arcs leading to blowout. The highest current point for each flow rate was governed by the maximum continuous power limit of the system $\left(750 \mathrm{~kW}_{\mathrm{e}}\right)$. Within this current range, arc-heater operation with hydrogen was found to be stable and repeatable even though EMI was quite intense. The load lines were found to be very linear over the operational range of interest with a drop in load impedance of about 100 volts when the flow rate was decreased by $1 \mathrm{~g} / \mathrm{s}$. This is intuitively understandable since a decrease in flow rate implies a corresponding reduction in chamber pressure for a fixed nozzle throat size, which leads to a lower plasma resistance at the same current level.

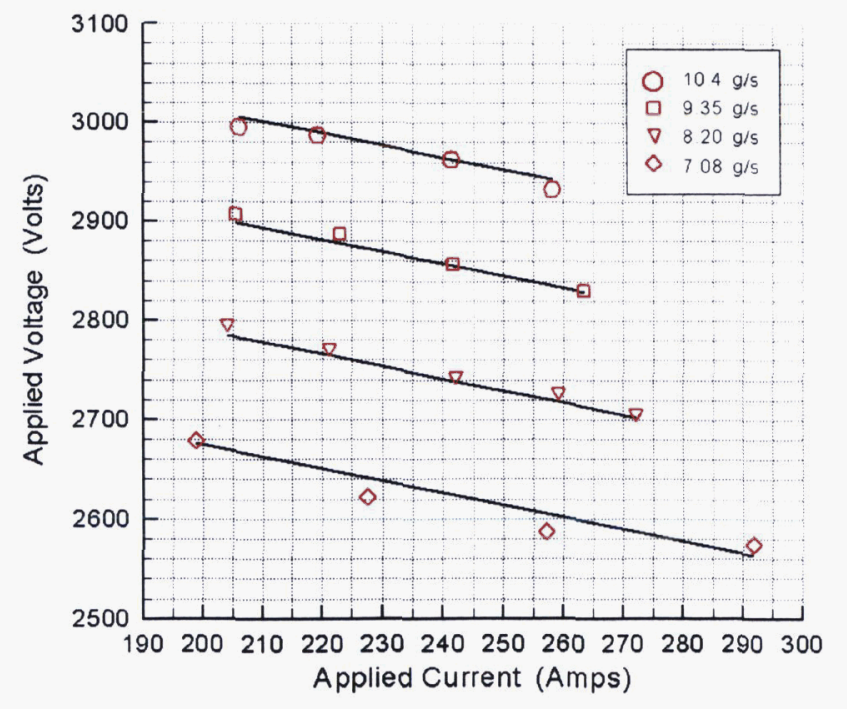

Figure 10. Arc-heater load lines at various hydrogen flow rates. 


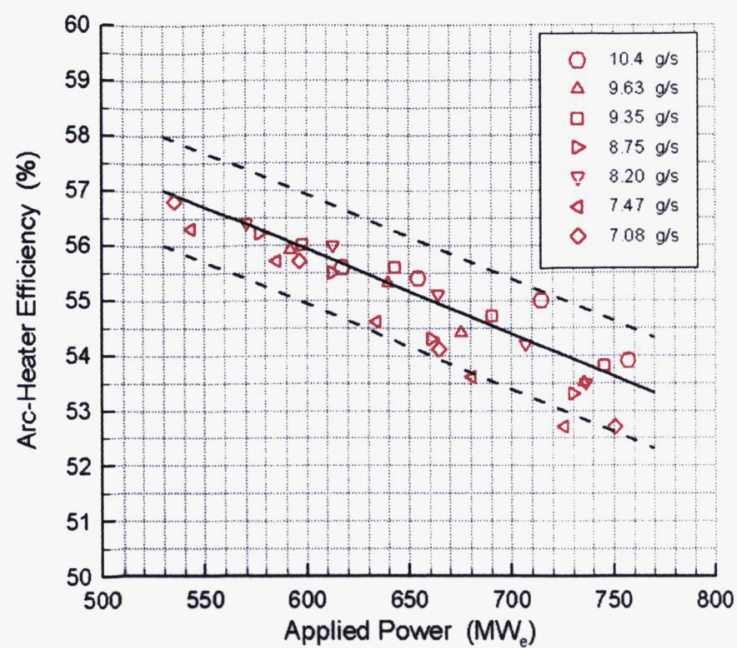

Figure 11. Inferred arc-heater efficiency shown as a function of applied power for hydrogen operation.

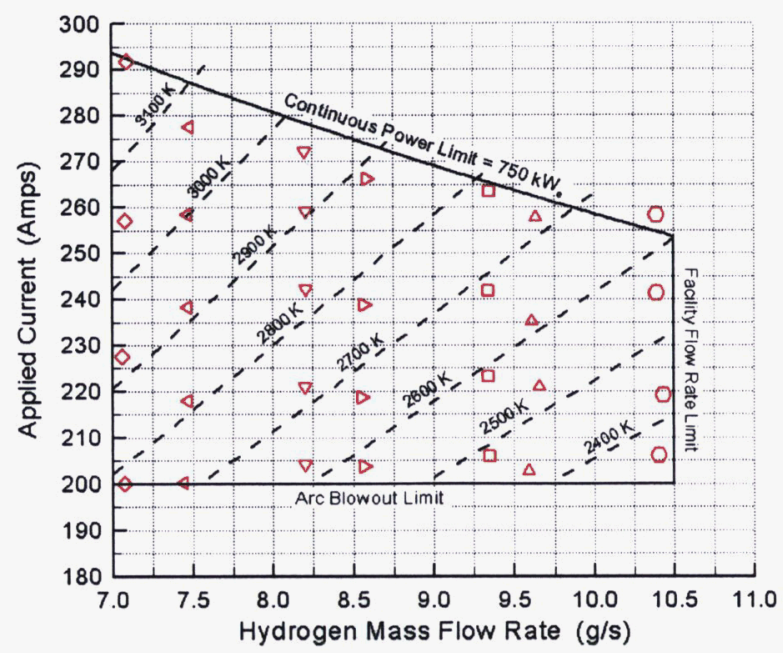

Figure 12. Arc-heater performance map as a function of applied current and hydrogen flow rate.

The arc-heater efficiency characteristics for hydrogen flow rates between 7.0 and $10.4 \mathrm{~g} / \mathrm{s}$ are summarized in Fig. 11 as a function of applied electrical power between 500 and $750 \mathrm{~kW}_{\mathrm{e}}$. It was observed that the inferred efficiencies could be correlated independently of flow rate with no more than a 1 percent deviation from the linear fit, as indicated by the dashed lines on Fig. 11. Generally speaking, the efficiency fell from about $57 \%$ at $550 \mathrm{~kW}_{\mathrm{e}}$ to approximately $54 \%$ at $750 \mathrm{~kW}_{\mathrm{e}}$. These results were based on a thermal power balance assuming that the globally measured heat loss power for the device accounted for all losses associated with the electrical-to-thermal energy conversion process.

The resulting arc-heater performance map is shown in Fig. 12 where the inferred chamber temperature has been correlated as a function of applied current and hydrogen mass flow rate. Symbols indicate data points used to construct the map, and the chamber temperature at each point was estimated by using the inferred thermal input power to define a pseudo heat of combustion for a NASA CEC based rocket calculation. Bounds on the operating range are indicated by solid lines on the map. The upper bound on applied current is determined by the maximum continuous power limit of the power supply $\left(750 \mathrm{~kW}_{\mathrm{e}}\right)$. The lower bound on applied current, on the other hand, is defined by arc stability considerations. That is, for currents below approximately $200 \mathrm{amps}$, the current density becomes too low to stabilize the arc and the discharge cannot be sustained within the flow rate range of interest. The upper bound on hydrogen mass flow rate is governed by basic facility throughput limitations, as established to meet test fixture design specifications. Within this bounded region, the accessible hydrogen temperature ranges from 2400 to $3100 \mathrm{~K}$, as needed to simulate NTR reactor environments. If less stressful start-up and shut-down transients are desired to avoid thermal shocking of material specimens, the temperature may be temporarily forced lower in the test fixture by introducing additional cold gas through four gas injection ports in the first expansion ring, as indicated in the layout of Fig. 5.

\section{Diagnostics Development}

\section{A. Raman Spectroscopy}

Detailed measurements of the hydrogen thermodynamic state and effects on NTR engine performance were of special interest in this project due to the limited quantity of reliable empirical data at high-pressure and hightemperature. To address this need, a laser-based Raman scattering technique was developed to measure the rotational temperature and number density of molecular hydrogen near the specimen test location. An independent measurement of total mass flow rate could then be used to infer dissociation fraction assuming local thermodynamic equilibrium. These measurements will prove useful for accurately defining environmental conditions during material exposure tests, anchoring CFD tools, and quantifying dissociation levels and resolving frozen flow effects on hydrogen rocket performance.

To facilitate development of the Raman diagnostic, our first step was to fabricate an exact mock-up of the test fixture which could be mated with an $\mathrm{H}_{2}$ /air Hencken Burner. Thus, excess hydrogen from the flame was available for diagnostic development in a controlled laboratory environment. The mock-up and layout of the components for laser excitation and signal for detection are shown in Fig. 13. This laboratory set-up was used extensively during the 
first year of the project as a means of developing and perfecting the laser-based spectroscopy technique. A major part of this effort included development of a methodology for generating theoretical $\mathrm{H}_{2}$ Raman spectra, which could be fitted to the detected scattering signals for determination of temperature. Some representative results from these bench top laboratory experiments are summarized in Fig. 14 showing the fit of the theoretical Raman spectra to the experimental spectra for different burner stoichiometries. Current project activity is directed toward implementation of this diagnostic technique in a practical field measurement system where the laser and detection optics are being duplicated in a climate-controlled portable building located adjacent to the hot hydrogen test apparatus. In the field measurement set-up, the laser beam will be transported out to the test fixture and the Raman scattering signal will be collected and transported back to a spectrometer equipped with an intensified CCD detector.

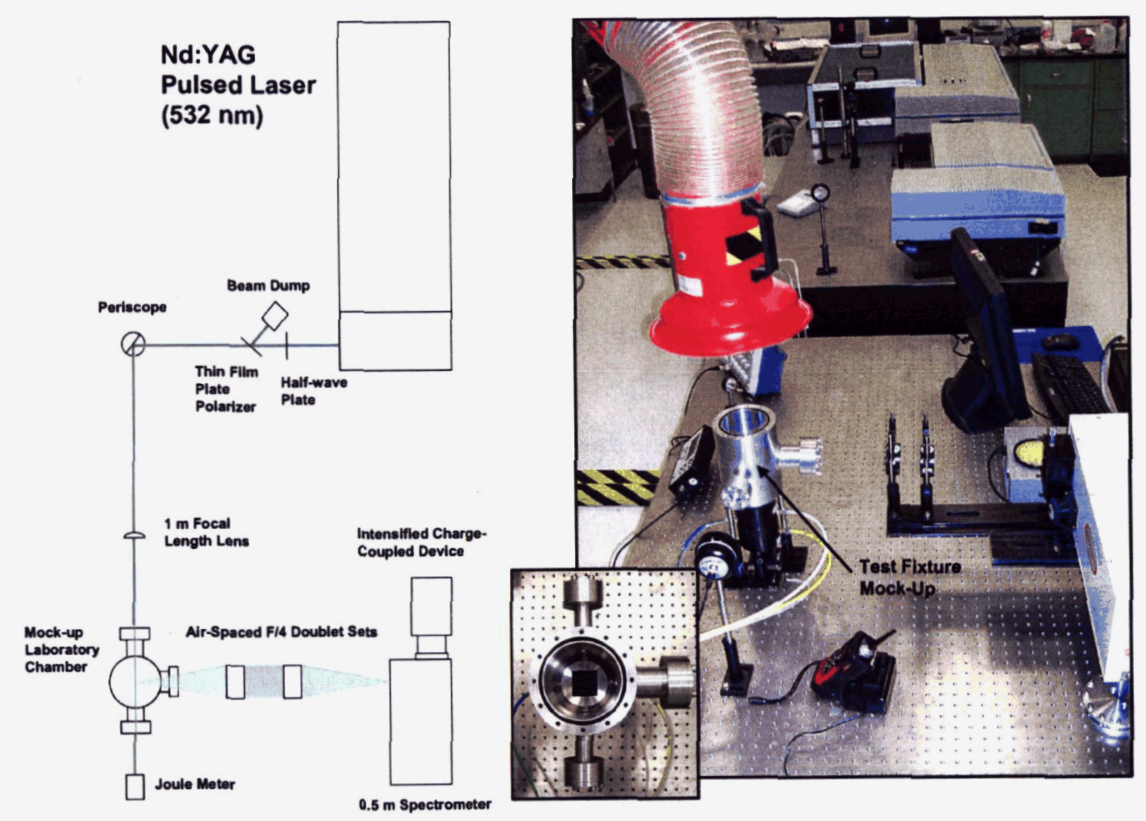

Figure 13. Bench top laboratory set-up of test fixture mock-up and Raman spectroscopy diagnostic.
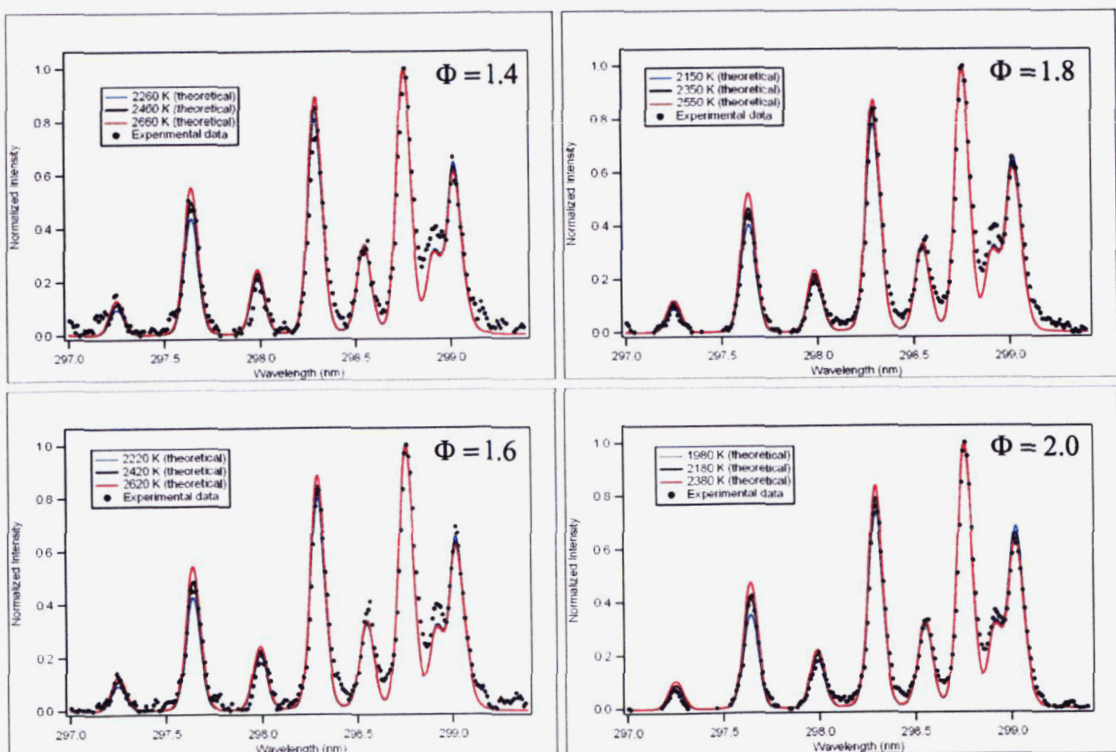

Figure 14. Fit of theoretical Raman spectra to experimental spectra for different burner stochiometries 


\section{B. Pyrometry}

Due to the dominance of radiant transport in high temperature environments and the known transparency of hydrogen, it would be very difficult to reliably infer specimen temperature without undertaking complicated conjugate heat transfer calculations, and a direct measurement approach is preferred. Thus, provisions were incorporated into the test fixture design which would permit monitoring of the specimen surface temperature using a commercially available optical pyrometer (Mikron Model MI-S140X). This particular model uses a single color bandpass at $677 \mathrm{~nm}$ with adjustable focal length from 19 to $42 \mathrm{~cm}$ and has a laser sighting mechanism for alignment. The temperature range is 1373 to $3773 \mathrm{~K}$. Temperature measurement requires independent knowledge of the spectral emissivity $\varepsilon(\lambda, T)$ at the target wavelength, but due to the strong temperature dependence of thermal radiance, the measurement is not very sensitive to this requirement. At most, the fractional temperature error is only 0.165 of the fractional emissivity uncertainty. Given the emissivity, the manufacturer specifies an accuracy of $0.5 \%$ for this instrument.

As a preliminary step, the pyrometer was set up and operated in a temporary test configuration to check out the data acquisition and to reconnoiter the measurement environment. For these tests, the pyrometer was bolted to the superstructure just upstream of the arc-heater exit plane and targeted on a 3/32-inch tungsten rod mounted transversely in the plume about 2.5 inches from the exit, as shown in Fig. 15. Temperatures were measured for a range of flow rates and discharge currents during test firings lasting about 30 seconds each. The temperatures were recorded with a nominal emissivity, but are easily adjusted in post-test analysis to account for any necessary emissivity corrections. It was found that electrical isolation of the instrument mount significantly reduced the noise level of the pyrometer output in the high EMI environment. Comparison of the pyrometer measurements with calculated stagnation temperatures indicated temperature predictions uniformly high by about 100 to $180 \mathrm{~K}$. The probable cause for this discrepancy is believed to be the deposition of carbon deposits on the tungsten rod as a result of methane after-burning in the plume, which increased the effective emissivity well above that of normal tungsten. Emissivity corrections for these surface deposits yielded average surface temperatures around $2500 \mathrm{~K}$ in good agreement with the calculated stagnation temperature. Actual exposure tests of NTR material specimens inside the test fixture will not be burdened with this complicating factor, and evidence thus far indicates that this pyrometry system will provide accurate and reliable surface temperature measurements.

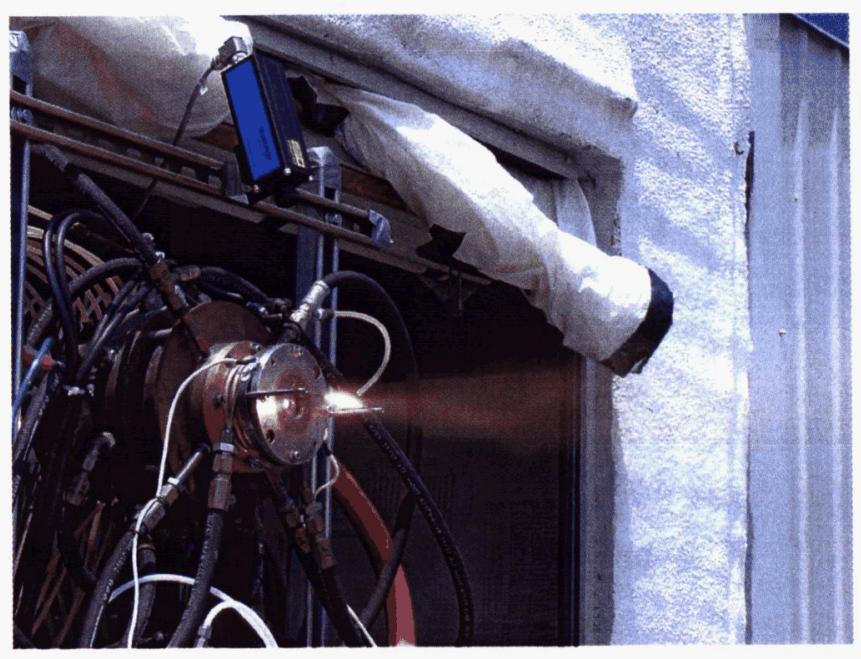

Figure 15. Temporary test configuration for pyrometry system check-out and environment reconnaissance.

\section{Material Specimen Processing \& Fabrication}

During the first year of project effort, processing techniques were developed to fabricate refractory metal and ceramic materials with a focus on the optimization of processes for CERMET and carbide materials such as W$\mathrm{Re} / \mathrm{UO} 2, \mathrm{~W}-\mathrm{Re} / \mathrm{UN},(\mathrm{U}, \mathrm{Zr}) \mathrm{C},(\mathrm{U}, \mathrm{Zr}, \mathrm{X}) \mathrm{C}$. The technical objective was to fabricate specimens for compatibility evaluation in the arc-heater test apparatus during the second year of the project. Special emphasis was placed on low-cost processing methods such as VPS and more conventional methods to fabricate near net shape parts. Following exposure to hot hydrogen flow, these specimens are to be carefully characterized in an attempt to optimize materials and processes leading to improved performance.

For the purpose of demonstrating basic operational capability and establishing baseline material characteristics, standard W-carbide and W/Re-carbide specimens were first fabricated to support initial testing efforts. A mounted $\mathrm{W}$-carbide specimen is shown in Fig. 7, for example. Identically sized tri-carbide specimens have also been fabricated with depleted uranium (DU) to support initial evaluation of mixed carbide fuels. These samples, which include four $(\mathrm{U}, \mathrm{Zr}, \mathrm{Nb}) \mathrm{C}$ specimens and four $(\mathrm{U}, \mathrm{Zr}, \mathrm{Ta}) \mathrm{C}$ specimens, are currently being characterized in a hydrogen furnace at the Institute for Space Nuclear Space Power \& Propulsion (INSPI) at the University of Florida. 
First year CERMET work was focused on evaluation of fabrication techniques to produce $\mathrm{W}$ and W-Re based materials with 20-60 volume percent $\mathrm{UN}$ or $\mathrm{UO}_{2}$ fuel particles. Conventional processing techniques including press/sinter and Hot Isostatic Pressing (HIP) were optimized to produce samples for hot hydrogen testing. Surrogate fuel particles such as $\mathrm{ZrN}$ and $\mathrm{HFN}$ have also been used to evaluate processing parameters such as particle size, composition, fuel loading, initial consolidation techniques, and sintering and HIP conditions.

Cold pressing and sintering is the simplest and most well known processing route for producing CERMET materials. For demonstration purposes, $\mathrm{W}-\mathrm{Re} / 20$ and 40 volume $\% \mathrm{ZrN}$ disc samples were pressed and sintered and $\mathrm{W}-\mathrm{Re} / 60$ volume $\% \mathrm{ZrN}$ rod sample were formed by CIP prior to sintering. The samples were fabricated using high purity elemental W, Re, and $\mathrm{ZrN}$ powders that were blended in a Turbula mixer. After blending, the powders were axial pressed at 35 and $60 \mathrm{ksi}$ into small samples using a standard punch and die set. The small disc samples allow quick evaluation of the blending and sintering parameters. Due to friction and non-uniform loading, only small samples are suited for axial pressing. Typically, CIP processes are used for consolidation of larger rod and tube sections. The samples were then sintered in both hydrogen and nitrogen at $2300^{\circ} \mathrm{C}$ to determine the effects on density, fuel stoichiometry, and alloying of the W-Re matrix. Based on the initial results, a second iteration of pressing and sintering was done using finer $\mathrm{W}$ and $\mathrm{ZrN}$ powder sizes. Following several trials, we were eventually able to achieve an as-sintered density of $80 \%$, but much longer sintering times will be required to achieve very high densities and complete alloying. For this reason, the focus of CERMET processing has shifted to direct HIP of blended powders with the use of a sacrificial can.

Vacuum Plasma Spray processes were also investigated for fabricating W-Re clad materials and tubular WRe/ZrN CERMET sections. The resulting VPS samples were approximately $90 \%$ dense with increased particle to particle bonding, which should significantly reduce sintering times. Both 20 and 40 volume $\% \mathrm{ZrN}$ cermets have also been fabricated by VPS. These samples were subsequently heat treated to further consolidate and alloy the materials. The current materials processing focus is directed at fabrication of $\mathrm{W}-\mathrm{Re} / \mathrm{ZrN}$ surrogate CERMET fuel specimens for future hot hydrogen exposure in the completed test fixture assembly.

\section{Conclusion}

An arc-heated NTR environments simulator has been developed which can generate hyper-thermal convective conditions traceable to reactor core conditions for low-cost, non-irradiated hot hydrogen exposure and screening of candidate fuel and structural materials. A water-cooled copper test fixture was designed and fabricated for exposure of 1 -inch long, $1 / 2$-inch diameter rod shaped material specimens in flowing hot hydrogen. The test fixture design also incorporates optical ports for real time optical pyrometry of specimen surface temperature and for implementation of a laser-based Raman spectroscopy diagnostic for measurement of molecular hydrogen rotational temperature and number density. Arc-heater performance mapping tests demonstrated an accessible temperature range of 2400 to $3100 \mathrm{~K}$ for hydrogen flow rates between 7 and $10.5 \mathrm{~g} / \mathrm{s}$.

Project efforts to date resulted in the establishment of a foundational hot hydrogen testing capability, and the stage is set for full utilization in support of materials development activities. Extensive laboratory development of the Raman spectroscopy diagnostic has transitioned to a practical field implementation stage, which should also yield a near-term capability for measurement of hot hydrogen thermodynamic properties in parallel with specimen/component testing. Immediate plans for the future are mainly directed at baseline specimen/component testing and demonstration of basic test capabilities. For example, the first series of specimen tests will be devoted to baseline testing of W-carbide and W/Re-carbide samples. During this time period, the HIP process will be further optimized using W-Re/ZrN and W-Re/HfN materials, and this knowledge will ultimately be used to fabricate surrogate CERMET fuel specimens for follow-on testing. As part of the test and evaluation program, standard metallography, scanning electron microscope (SEM), and x-ray diffraction (XRD) techniques will be used to characterize all materials in as-fabricated and post-test conditions.

Envisioned facility upgrades and improvements include development of an exhaust filtration system which would permit exposure of Depleted Uranium (DU) containing material specimens. Preliminary analysis and design work for this upgrade has been completed, and a working system could be rapidly completed and installed as the need arises. Additional design work is also underway for a new test fixture module which incorporates an electrical resistive element for backside heating of a tubular material specimen with internally flowing hydrogen thus providing a more realistic simulation of thermal gradients in the specimen.

\section{Acknowledgments}

This work was conducted under the auspices of MSFC Focus Area IR\&D Project 2005-80 entitled "Hot Hydrogen Materials and Component Development." 